\title{
AJUSTE DE UNA FUNCIÓN DE PRODUCCIÓN AL SECTOR FINANCIERO EN COLOMBIA*
}

\author{
ALEJANDRO RAMÍREZ VIGOYA** \\ UNIVERSIDAD MILITAR NUEVA GRANADA
}

Recibido/ Received/ Recebido: 18/03/2014 - Aceptado/ Accepted / Aprovado: 29/08/2014

\begin{abstract}
Resumen
Este trabajo desarrolla en forma pormenorizada la metodología de datos panel para encontrar la mejor especificación econométrica de una función de producción, al comparar la Cobb-Douglas y la translogarítmica con datos del sector financiero colombiano. Se encuentra que la función que mejor se ajusta es la translogarítmica con efectos fijos, lo que indica que, por un lado, existen diferencias significativas en los retornos del capital y del trabajo sobre los ingresos entre bancos y, por otro lado, que existen efectos de segundo orden en el sector y que las elasticidades son variables. Además, se encuentra que el capital y el trabajo tienen productividades marginales decrecientes y estos dos factores son complementarios. Por otro lado, se demuestra que la elasticidad del capital es unitaria y significativa, mientras que el factor trabajo solo complementa al capital, siendo más una herramienta que un aporte significativo para el ingreso de los bancos.
\end{abstract}

Palabras clave: Modelos de datos panel, Función Cobb-Douglas, Función translogarítmica, Elasticidades, Efectos de segundo orden.

\section{SETTING OF A PRODUCTION FUNCTION TO THE FINANCIAL SECTOR IN COLOMBIA}

\begin{abstract}
This work develops in detail the methodology of panel data to find the best econometric specifications of a production function, comparing the Cobb-Douglas and the translog with data from the Colombian finance sector. It's found that the function that adjusts better is the translog with fixed effects, which indicates, on one hand, that there are a significant difference in relation to capital return and the work on the income between banks; and for another hand, that there are secondary effects in the sector and elasticities vary. Also, it's found that the capital and work have decreasing marginal productivities and that these two factors are complementary. From another side, it's demonstrated that elasticity of capital is unitary and significant; meanwhile the work factor only complements to capital, being more a tool than a significant contribution for the banks income.

Keywords: Panel data models, Cobb-Douglas Function, Translog function, Elasticities, Second order effects.

Este es un artículo de investigación que pertenece al proyecto: "Un análisis estructural de la política monetaria en Colombia" (ECO970), adscrito a la línea de investigación sobre políticas económicas del Grupo de Investigación CIE. Financiado por la Vicerrectoría de Investigaciones de la Universidad Militar Nueva Granada.

**: Profesor de planta y economista de la Universidad Militar Nueva Granada. Correo electrónico: alejandro.ramirez@unimilitar.edu.co, alej-ram@uniandes.edu.co. Tel: (57) (1) 6500000 Ext. 1306. Dirección postal: Cra. 11 \# 101-80, Bloque C, piso 1, Centro de Investigaciones Económicas, Bogotá (Colombia).
\end{abstract}




\title{
AJUSTE DE UMA FUNÇÃO DE PRODUÇÃO AO SETOR FINANCEIRO NA COLÔMBIA
}

\begin{abstract}
Resumo
Este trabalho desenvolve em forma pormenorizada a metodologia de dados de painel para encontrar a melhor especificação econométrica de uma função de produção, ao comparar a CobbDouglas e a translogarítmica com dados do setor financeiro colombiano. Encontra-se que a função que melhor se ajusta é a translogarítmica com efeitos fixos, o que indica que, por um lado, existem diferenças significativas nos retornos do capital e do trabalho sobre os rendimentos entre bancos $e$, por outro lado, que existem efeitos de segunda ordem no setor e que as elasticidades são variáveis. Além disto, encontra-se que o capital e o trabalho têm produtividades marginais decrescentes e estes dois fatores são complementares. Por outro lado, demonstra-se que a elasticidade do capital é unitária e significativa, enquanto o fator trabalho só complementa ao capital, sendo mais uma ferramenta que uma contribuição significativa para o rendimento dos bancos.

Palavras chave: Modelos de dados de painel, Função Cobb-Douglas, Função translogarítmica, Elasticidades, Efeitos de segunda ordem.

Ramírez, A. (2015). Ajuste de una función de producción al sector financiero en Colombia. En: Revista de la Facultad de Ciencias Económicas de la Universidad Militar Nueva Granada. rev.fac. cienc.econ, XXIII (1).

JEL: C52, D01, G21.
\end{abstract}

\section{Introducción}

En el sector financiero se han estudiado, bajo diferentes perspectivas, los retornos de los factores de producción sobre los ingresos.

Los trabajos de investigación que se enfocan en la eficiencia del sector financiero utilizan diferentes metodologías que generalmente parten de una única especificación de la función de producción. Sin embargo, no es común encontrar en la literatura trabajos que comparen diferentes formas funcionales que la función de producción podría tomar, dependiendo de los datos reales de un sector específico de la economía.

Una de las metodologías utilizadas para medir la eficiencia es crear un índice que mide la eficiencia en la intermediación financiera (Server \& Melián, 2001), en este caso, el índice hace posible le medición de la eficiencia y la evaluación de ésta en un periodo dado.

Otro tipo de trabajos se enfocan en la relación beneficio costo de la utilización de capital financiero sobre la eficiencia en el sistema bancario, como el de Estrada \& Osorio (2004). En éste trabajo se llega a la conclusión de que es posible que exista poder de mercado en el sector financiero colombiano, lo cual se traduciría en rentas monopólicas.

Por el lado de las formas específicas que toman las funciones que miden los retornos de los factores de producción, la teoría económica aporta una serie de aproximaciones que explican las relaciones tecnológicas entre los factores y el producto final. Un grupo de funciones hacen suposiciones sobre las elasticidades de sustitución y sobre los retornos a escala, como son las funciones Cobb-Douglas, que suponen una elasticidad de sustitución igual a uno y efectos marginales de primer orden.

Dentro de los trabajos enfocados en elasticidades de sustitución constantes, es decir, con curvaturas constantes de la función de producción, como la función Cobb-Douglas, el trabajo de Humphrey (2001) hace interesantes análisis sobre el álgebra de la funciones de producción cuando se utilizan estas funciones, y 
específicamente sobre las elasticidades de sustitución y sobre los retornos a escala, en las cuales se supone una elasticidad de sustitución es igual a uno.

A nivel internacional está el trabajo de Arrow et al. (1961), en el cual se analiza tanto la eficiencia en el uso de los factores de producción como el grado de sustituibilidad del capital y el trabajo.

Por otro lado están las funciones CES (Constant Elasticity Sustitution), que relaja la suposición de elasticidad igual a uno y, permite que la elasticidad de sustitución varíe entre menos uno e infinito. Las funciones CES abarcan a las Cobb-Douglas, Leontief y lineales, dependiendo del valor de la elasticidad de sustitución que cada una tome.

Un trabajo interesante en este tema es el de Fletcher \& Lu (1968), en el cual se aplica una función CES generalizada. En el trabajo de Klump \& Freissler (2000), se analizan las controversias e inconsistencias al utilizar las funciones de producción CES en modelos de crecimiento. En el grupo de trabajos que comparan funciones de producción CES y CobbDouglas está el de Brown (1970), quien examina funciones de producción bajo el supuesto de que la empresa busca minimizar costos para unos precios y output dados.

Otro grupo de formas funcionales son más flexibles en cuanto a las suposiciones sobre las elasticidades de sustitución y los retornos a escala. En este grupo se encuentra la función de producción trascendental logarítmica (translog) y la función de producción de elasticidad de producción variable (VES). En el primer caso está el trabajo de Christensen et al. (1973), el cual se enfoca en las fronteras de producción de las funciones trascendentales logarítmicas y su aplicación para analizar las fronteras tecnológicas de los factores de producción.

Un trabajo interesante que utiliza las funciones translogarítmicas es el de Kim (1992), quien demuestra utilizando funciones inversas de demanda, que, para el sector manufacturero de Estados Unidos, la tecnología de producción es no homotética. También utilizando funciones de producción translog está el trabajo de Burras \& Moroney (1975), en el cual se analiza la elasticidad de sustitución entre el capital, el trabajo y los recursos naturales en el sector manufacturero de Estados Unidos.

Hay interesantes trabajos que utilizan y comparan tres formas funcionales: Cobb-Douglas, CES y translog, dentro de los cuales está el trabajo de Mefford (1986), en el cual se comprueba que las funciones cumplan con las condiciones de consistencia, monotonicidad y cuasi-concavidad en el sector de manufacturas de bienes de consumo.

Sobre la aproximación de la tecnología en formas funcionales, la teoría económica aporta una serie de aproximaciones que explican las relaciones tecnológicas entre los factores y el producto final, tal es el caso de los trabajos de Kraipornsak (2001), Ryu \& Zellner (1998) y Walters (1963), en los cuales se plantea la diferencia entre las funciones de producción estimadas bajo diferentes metodologías econométricas, así como las diferentes especificaciones de las funciones de costos y de los retornos de escala.

En el presente trabajo se analizarán los retornos de los factores (trabajo y capital como input) sobre los ingresos (output), comparando dos modelos: el Cobb-Douglas, que supone elasticidad de sustitución y retornos constantes y el translogarítmico (translog) que supone elasticidad de sustitución y retornos variables.

El primer modelo a utilizar es la conocida función Cobb-Douglas, en la cual los retornos de los factores suponen que la elasticidad de sustitución entre éstos es igual a uno. Por otro lado, los coeficientes del modelo, en este caso son las elasticidades del capital y del trabajo, son constantes. Este modelo supone que no existen efectos de segundo orden, sino solamente efectos de primer orden, que son las elasticidades. Suponer que solamente existen primeras derivadas es bastante restrictivo y no real para los datos, por lo tanto en este trabajo se contrasta esta suposición con la existencia de efectos de segundo orden.

El segundo modelo a utilizar es una función de producción translogarítmica. Esta aproximación econométrica supone que existen efectos de segundo orden entre los factores de producción (input) y la 
producción final (output), por lo que las elasticidades son variables. En otras palabras, se puede suponer que existen segundas derivadas en la función de producción, lo que se traduce en la existencia de efectos marginales crecientes o decrecientes. A partir de la función translogarítmica también se puede encontrar la relación de sustitución (o de complementariedad) entre los factores de producción.

Los créditos bancarios que son los principales activos de los bancos, se utilizan como variable capital, que es uno de los factores de producción que explican al output en el sector financiero.

Como variable trabajo se utilizará una proxy ${ }^{1}$, los salarios y honorarios de los empleados, que en muchos trabajos empíricos están correlacionados con el número de trabajadores de la empresa. Como variable dependiente se utilizan los ingresos e intereses percibidos por los bancos en cada periodo de tiempo. Para los dos modelos se utilizan las mismas variables explicadas anteriormente.

El principal objetivo del trabajo es buscar la mejor especificación econométrica en modelos de datos panel comparando los modelos sin efectos, con efectos fijos y con efectos aleatorios. Un segundo objetivo es comparar estadísticamente las especificaciones de los modelos Cobb-Douglas y translogarítmico, para saber cuál de los dos se ajusta mejor a los datos del sector financiero en Colombia. Por último, de acuerdo a la correcta forma funcional encontrada se encontrarán las verdaderas elasticidades del capital, del trabajo y de escala en el sector financiero colombiano.

\section{Datos}

Para este trabajo se utilizaron como muestra los datos desde 1995 hasta 2008 del PyG ${ }^{2}$ de los bancos comerciales en Colombia (ABN Amrobank, Banco de Colombia, Banco de Bogotá, Banco de Crédito (Helms Bank), Banco Popular, Banco Bilbao Vizcaya Argentaria, Citibank, Hongkong and Shanghai Banking Corporation (HSBC), Banco Santander, Banco AV Villas, Banco Caja Social Colmena BCSC, Banco de Occidente, Banco Sudameris), los datos del Banco Colpatria son desde 1998 y los del Banco Agrario desde $1999^{3}$.

El Banco Santander se retiró de Colombia en 2012 pero nuevamente retorna en el 2014 en búsqueda de un nicho de mercado más internacional. En 2005 el Banco Sudameris se fusiona con el Banco Tequendama para crear el banco GNB Sudameris, el cual compra en el 2012 al Banco HSBC en Colombia, Perú, Uruguay y Paraguay para crear el nuevo Banco GNB.

Por otro lado, para medir la eficiencia en el sector financiero las variables utilizadas en este trabajo son: ingresos e intereses como variable explicada u output. Los inputs utilizados son los préstamos como el factor capital y los sueldos y honorarios como una aproximación del factor trabajo. Esta última variable se emplea como una proxy del número de trabajadores.

Para correr los modelos econométricos es necesario que los datos de las muestras estén en términos reales o constantes, por lo tanto los datos obtenidos fueron deflactados con base $1998^{4}$.

\section{Especificación matemática}

Los fundamentos teórico-matemáticos utilizados en este trabajo, se centran en la utilización de dos modelos utilizados frecuentemente en funciones de producción y de costos, el Cobb-Douglas y el translogarítmico. En este trabajo se utilizan para medir los retornos de los factores (capital y trabajo) sobre el ingreso, en el sector financiero.

Una variable proxy está altamente correlacionada con la variable que reemplaza. En este caso los sueldos se supone están correlacionados con el número de trabajadores.

Estado de pérdidas y ganancias.

Esta información fue recopilada de la Superintendencia Financiera.

El cálculo de las regresiones se pueden tomar con todos los datos en términos nominales (corrientes), o con todos los datos en términos reales (constantes). Los resultados econométricos no cambian por las propiedades de las funciones lineales. 


\subsection{Función Cobb-Douglas}

Matemáticamente la función Cobb-Douglas tiene la siguiente especificación:

$$
Y=A K^{\alpha} L^{\beta}
$$

Donde $Y$ es el output que se requiera explicar según el modelo, la constante A mide el nivel tecnológico o la productividad de los factores de producción, K es el stock de capital físico, L es el trabajo (K y L representan los factores de producción conocidos también como los inputs). Por otro lado $\alpha$ es la elasticidad del stock de capital y $\beta$ es la elasticidad del trabajo.

A continuación se presenta la deducción matemática de las elasticidades del capital y del trabajo.

La elasticidad del capital se define como:

$$
\varepsilon_{K}=\partial Y / \partial K * K / Y=\frac{\alpha A K^{\alpha-1} L^{\beta} K}{A K^{\alpha} L^{\beta}}=\alpha
$$

La elasticidad del trabajo se define como:

$$
\varepsilon_{L}=\partial Y / \partial L * L / Y=\frac{\beta A K^{\alpha} L^{\beta-1} L}{A K^{\alpha} L^{\beta}}=\beta
$$

Por lo anterior se deduce que si el modelo correcto es el Cobb-Douglas, entonces las elasticidades son constantes.

La aproximación matemática de la función CobbDouglas en este trabajo, parte de la linealización de (1), que resulta al aplicar logaritmos naturales en (1). Surge entonces la expresión (2) que es más manejable tanto matemática como econométricamente.

$$
\mathrm{LnY}=\ln \mathrm{A}+\alpha \ln \mathrm{K}+\beta \ln \mathrm{L}
$$

Donde:

$\mathrm{LnY}=$ es el logaritmo natural de los ingresos e intereses de los bancos.

LnK = es el logaritmo natural de los activos de los bancos, en este trabajo los préstamos son considerados como los activos.

$\mathrm{LnL}=$ es el logaritmo natural de los sueldos y honorarios de los trabajadores. $\rho_{0}=$ es la constante del modelo,

$\alpha=\quad$ es la elasticidad de los ingresos de los bancos con respecto a los activos de los mismos y

$\beta=$ es la elasticidad de los ingresos de los bancos con respecto a los sueldos y honorarios de los trabajadores.

\subsection{Función translogarítmica}

La función translogarítmica es más general que la Cobb-Douglas, siendo esta última una forma específica de la primera. La forma matemática de la función translogarítmica es la siguiente:

$$
\operatorname{Ln} Y=\ln A+\alpha \ln K+\beta \ln L+\theta\left(1 / 2 \ln ^{2} K\right)+\eta\left(1 / 2 \ln ^{2} L\right)+\sigma \ln K \ln L
$$

El modelo translogarítmico en (3) incluye a la función Cobb-Douglas en (2), la diferencia es que en esta última no hay efectos de segundo orden, es decir, segundas derivadas. El modelo translogarítmico en (3) permite saber si las productividades marginales son crecientes o decrecientes y también permite conocer la relación existente entre los factores de producción, es decir, si son complementarios o sustitutos. La función de producción translogarítmica no supone elasticidad de sustitución constante como la Cobb-Douglas, para la cual la elasticidad de sustitución es igual a uno.

En la función translogarítmica las elasticidades del capital y del trabajo son respectivamente:

$$
\varepsilon_{K}=\frac{\partial \ln Y}{\partial \ln K}=\frac{\dot{Y} / Y}{\dot{K} / K}=\alpha+\theta \ln K+\sigma \ln L
$$

$$
\varepsilon_{L}=\frac{\partial \ln Y}{\partial \ln L}=\frac{\dot{Y} / Y}{\dot{L} / L}=\alpha+\eta \ln L+\sigma \ln K
$$

Por (4) y (5) se puede deducir que si el modelo correcto es el translogarítmico, entonces las elasticidades son variables, dependiendo de los valores de capital y trabajo con los cuales se midan. Generalmente para estos valores se toman las medias de las variables. 
La elasticidad de escala se define como:

$$
\varepsilon_{\text {esc }}=\varepsilon_{K}+\varepsilon_{L}
$$

Y las segundas derivadas son:

$$
\begin{aligned}
& \varepsilon_{K K}=\frac{\partial \varepsilon_{K}}{\partial \ln K}=\theta \\
& \varepsilon_{L L}=\frac{\partial \varepsilon_{L}}{\partial \ln L}=\eta \\
& \varepsilon_{K L}=\varepsilon_{L K}=\frac{\partial \varepsilon_{K}}{\partial \ln L}=\frac{\partial \varepsilon_{L}}{\partial \ln K}=\sigma
\end{aligned}
$$

Estadísticamente se puede comprobar que si $\theta, \eta$ y $\sigma$ son cero en (2), entonces la función correcta a utilizar es la Cobb-Douglas en (1) y si estos parámetros no son cero, entonces la función correcta es la translogarítmica.

Si la función correcta es la translogarítmica entonces, si $\theta$ y $\eta$ son positivas entonces hay rendimientos marginales crecientes, si son negativas entonces hay rendimientos marginales decrecientes.

Por otro lado, si la función correcta es la translogarítmica, entonces el signo del parámetro $\sigma$ en (2) nos indica la relación entre el capital y el trabajo. Si es positivo entonces el capital y el trabajo son complementarios, por el contrario, si es negativo entonces el capital y el trabajo son sustitutos.

\section{Especificación econométrica}

Dado que lo datos son tanto de corte transversal como de series de tiempo, el modelo a correr es de datos panel. Bajo la especificación de datos panel, Greene (2001) estable que hay tres posibilidades: un modelo sin efectos, un modelo con efectos fijos o un modelo con efectos aleatorios.

Un modelo econométrico sin efectos se corre por mínimos cuadrados ordinarios (MCO) como una regresión lineal simple y el modelo supone que no hay diferencias significativas entre las empresas, por lo que la constante es la misma para todas las empresas.
En el modelo con efectos fijos se incluyen variables dummy por empresa o por agente y también se corre con mínimos cuadrados ordinarios (MCO). Mientras que el modelo con efectos aleatorios no se incluyen las dummies y además se corre por el método de mínimos cuadrados generalizados factibles (MCGF).

Como el modelo (3) incluye al modelo (2), entonces econométricamente lo que se hace es encontrar la mejor especificación del modelo translogarítmico en (3), para luego estadísticamente demostrar si éste o el Cobb-Douglas es la función que mejor se acondiciona a los datos del sector financiero. Para ello se presentan las tres especificaciones del modelo translogarítmico: sin efectos, con efectos fijos y con efectos aleatorios.

La especificación econométrica de una función translogarítmica fue utilizada por primera vez por Christensen et al. (1973). La especificación econométrica sin efectos es la siøuiente:

$$
\begin{gathered}
\operatorname{Ln} Y_{i t}=\mu_{0}+\mu_{1} \ln K_{i t}+\mu_{2} \ln L_{i t}+\mu_{3}\left(1 / 2 \ln ^{2} K_{i t}\right)+ \\
\mu_{4}\left(1 / 2 \ln ^{2} L_{i t}\right)+\mu_{5} \ln K_{i t} \ln L_{i t}+\varepsilon_{i t}
\end{gathered}
$$

El modelo con efectos fijos es el que sigue:

$$
\begin{aligned}
\operatorname{Ln} Y_{i t}= & \alpha_{0}+\alpha_{i}+\alpha_{1} \ln K_{i t}+\alpha_{2} \ln L_{i t}+\alpha_{3}\left(1 / 2 \ln ^{2} K_{i t}\right)+ \\
& \alpha_{4}\left(1 / 2^{2} \ln ^{2} L_{i t}\right)+\alpha_{5} \ln K_{i t} \ln L_{i t}+\varepsilon_{i t}
\end{aligned}
$$

En el modelo (10) el subíndice i representa al i-ésimo banco y el subíndice t representa el t-ésimo año. En el modelo (10) hay diferencias en la constante según el banco. Pero si se comprueba que no hay variaciones significativas entre bancos en el intercepto, entonces el modelo que se debería correr es el de efectos aleatorios, cuya especificación es:

$$
\begin{gathered}
\operatorname{Ln} Y_{i t}=\beta_{0}+\beta_{1} \ln K_{i t}+\beta_{2} \ln L_{i t}+\beta_{3}\left(1 / 2 \ln ^{2} K_{i t}\right)+ \\
\beta_{4}\left(1 / 2 \ln ^{2} L_{i t}\right)+\beta_{5} \ln K_{i t} \ln L_{i t}+\varepsilon_{i t}
\end{gathered}
$$

En la especificación (11) la constante $\beta_{0}$ es un solo valor que no cambia por banco. El paso a seguir es hacer las pruebas de qué modelo se corre, el (9), el 
(10) o el (11), para luego hacer la contrastación de si la especificación elegida es la translogarítmica o la Cobb-Douglas.

\subsection{Modelo con efectos fijos vs modelo sin efectos}

El primer paso para saber qué modelo elegir entre el (9) o el (10) es correr estos modelos y calcular la siguiente prueba $\mathrm{F}$ :

$$
F(n-1, n T-n-K)=\frac{\left(R_{U}^{2}-R_{P}^{2}\right) /(n-1)}{\left(1-R_{U}^{2}\right) /(n T-n-K)}
$$

Donde $R_{U}^{2}$ es el $\mathrm{R}$ cuadrado de la regresión con efectos fijos (10), y $R_{P}^{2}$ es el $\mathrm{R}$ cuadrado de la regresión sin efectos (9). En este caso la función no restringida es la de efectos fijos y la restringida es la que no tiene efectos por empresa.

Las hipótesis son:

Ho: modelo sin efectos fijos

Ha: modelo con efectos fijos

Las regresiones de los modelos (9) y (10) se presentan en los Anexos 1 y 2 . Con los resultados de las regresiones el valor calculado de la $\mathrm{F}$ es 30,74 con una probabilidad de 0,0000: los resultados de esta prueba se presentan en el Anexo 3. Por lo tanto se aprueba la hipótesis alterna, es decir, entre estos dos modelos se elige el de efectos fijos.

Una vez escogido el modelo de efectos fijos, el siguiente paso es elegir entre el modelo de efectos fijos y el modelo de efectos aleatorios.

\subsection{Efectos fijos vs efectos aleatorios}

Las dos pruebas principales para el contraste de efectos fijos versus efectos aleatorios son la de Breusch-Pagan o de Multiplicadores de Lagrange y la de Hausman, que es la que más comúnmente se calcula.
Para la prueba de Breusch-Pagan se calcula el estadístico en (13) y luego se compara con una Chi cuadrado con un grado de libertad.

$$
M L=\frac{n T}{2(T-1)}\left[\frac{\sum_{i=1}^{n}\left[\sum_{t=1}^{T} e_{i t}\right]^{2}}{\sum_{i=1}^{n} \sum_{t=1}^{T} e_{i t}^{2}}-1\right]^{2}
$$

Donde $e_{i t}$ son los residuos del modelo (9), es decir, el modelo sin efectos cuyos resultados están en el Anexo 1. Los resultados de la prueba de Breusch-Pagan se presentan en el Anexo 4.

Por otro lado la prueba de Hausman consiste en calcular un estadístico de Wald con la fórmula (14), que se compara con una Chi cuadrado con un grado de libertad o también se le puede calcular la raíz cuadrada a (14) y se compara con la distribución normal.

$$
W=N T \hat{q}^{\prime}[\operatorname{Var}(\hat{q})]^{-1} \hat{q}
$$

Donde:

$$
\begin{aligned}
& \hat{q}=\hat{\beta}_{E F}-\hat{\beta}_{E A} \\
& \operatorname{Var}(\hat{q})=\operatorname{Var}\left(\hat{\beta}_{E F}\right)-\operatorname{Var}\left(\hat{\beta}_{E A}\right)
\end{aligned}
$$

En (15) $\hat{\beta}_{E F}$ es el vector de coeficientes del modelo con efectos fijos excluyendo a las variables dicotómicas (Anexo 2) y $\hat{\beta}_{E A}$ es el vector de coeficientes del modelo con efectos aleatorios (Anexo 3).

Por otro lado, $\operatorname{Var}\left(\hat{\beta}_{E F}\right)$ es la matriz de varianzas y covarianzas del modelo con efectos fijos excluyendo a las variables dicotómicas y $\operatorname{Var}\left(\hat{\beta}_{E A}\right)$ es la matriz de varianzas y covarianzas del modelo de efectos aleatorios.

Los resultados de las pruebas en (13) y (14) se presentan en la Tabla 1.

En las dos pruebas la hipótesis nula es que el modelo correcto es el de efectos aleatorios y la hipótesis alterna es que el modelo correcto es el de efectos 
Tabla 1. Contrastes de especificación: efectos fijos versus efectos aleatorios ${ }^{5}$

\begin{tabular}{|c|c|c|c|c|}
\hline Prueba & Estadístico & Probabilidad & Significancia & Modelo \\
\hline Breusch-Pagan & 345,04 & 0,0000 & 0,95 & Ha: Efectos fijos \\
\hline Hausman & 9,8 & 0,0811 & 0,9 & Ha: Efectos fijos \\
\hline
\end{tabular}

fijos. Con la prueba de Breusch-Pagan se aprueba la hipótesis alterna con el $95 \%$ de confiabilidad, que en este caso es el modelo con efectos fijos. Con la prueba de Hausman también se aprueba el modelo de efectos fijos, pero la significancia es del $90 \%$. Entonces el modelo correcto en datos panel para correr es el (10).

La prueba de Breusch-Pagan es más robusta que la prueba de Hausman, sin embargo, la de Hausman aprueba con un $90 \%$ de confiabilidad al modelo de efectos fijos.

Por otro lado, el modelo (10) se corrió con errores robustos para evitar los problemas de heterocedasticidad $^{6}$. Lo que en últimas se hace econométricamente es una corrección robusta de la matriz de varianzas y covarianzas.

\subsection{Modelo translogarítmico}

Los resultados del modelo (10) con efectos fijos se presentan en la tabla 2 , que es un resumen del Anexo 2.

En los resultados de la Tabla 2 la constante corresponde al parámetro del ABN Amro Bank. La cuestión es que en stata o en cualquier paquete econométrico una de las dummies se elimina y es reemplazada por la constante. Entonces, en este caso, la dummy que se eliminó fue la del banco ABN y la constante es el parámetro de este banco. Si no se eliminara una de las dummies, entonces habría colinealidad perfecta entre las trece dummies y la constante y la regresión tendría problemas de colinealidad.
Para saber que banco tiene mayores retornos de los inputs (capital y el trabajo) sobre el output (ingresos) entonces a la constante, que en este caso es el Amro Bank se le suma cada uno de los coeficientes de los otros bancos. En este caso el parámetro para comparar es el del Amro Bank. Por ejemplo, para saber el parámetro del Banco de Occidente se suma el parámetro del Amro Bank más el parámetro del Banco de Occidente; la suma da 2,6582.

Al hacer esta operación con todos los bancos resulta que el banco con mayores retornos del capital y el trabajo (mayor desplazamiento de la constante) es el Banco de Occidente y el de menores retornos es precisamente el Amro Bank.

\subsection{Contraste de especificación Cobb-Douglas vs. translogarítmico}

Dado que se eligió el modelo translogarítmico (3), cuyos resultados están en la Tabla 2 , y que además incluye a la especificación Cobb-Douglas (2), el siguiente paso es demostrar si se cumple en (10), que $\alpha_{3}, \alpha_{4}$ y $\alpha_{5}$ son todos iguales a cero, o lo que es lo mismo que los coeficientes en la tabla 2 de $\ln ^{2} \mathrm{~K}, \ln ^{2} \mathrm{~L}$ y $\operatorname{lnK} * \ln L$ son todos iguales a cero, como hipótesis nula, o si no son iguales acero, como hipótesis alterna. Si se demuestra que todos los coeficientes son cero, entonces el modelo a analizar es el Cobb-Douglas, de lo contrario es el translogarítmico.

En la literatura econométrica hay varias formas de contrastar la hipótesis de que el modelo correcto es el Cobb-Douglas (2) frente a la hipótesis de que el modelo correcto es el translogarítmico (3).

\footnotetext{
Fuente: Elaboración propia en Stata.

6 En el Anexo 2 se presenta la salida en Stata de la regresión de datos panel con efectos aleatorios y corregidos con errores robustos.
} 
Tabla 2. Modelo Translogarítmico con Efectos Fijos corregido por errores estándar robustos

\begin{tabular}{|c|c|c|c|}
\hline & Coeficiente & $\begin{array}{c}\text { Errores Estan. } \\
\text { Robustos }\end{array}$ & p-value \\
\hline LnK & 0,958373 & 0,2251422 & 0,000 \\
\hline $\operatorname{lnL}$ & $-0,274348$ & 0,2163843 & 0,207 \\
\hline $\ln 2 \mathrm{~K}$ & $-0,074974$ & 0,0375462 & 0,047 \\
\hline Ln2L & $-0,063809$ & 0,0418219 & 0,129 \\
\hline $\operatorname{lnK} K^{*} \ln L$ & 0,079394 & 0,0345116 & 0,023 \\
\hline Bancolombia & 0,320402 & 0,0556479 & 0,000 \\
\hline Banco de Bogotá & 0,288434 & 0,0527660 & 0,000 \\
\hline Banco de Crédito & 0,185176 & 0,0463744 & 0,000 \\
\hline Banco Popular & 0,259225 & 0,0520588 & 0,000 \\
\hline BBVA & 0,344738 & 0,0539656 & 0,000 \\
\hline Citibank & 0,256667 & 0,0515412 & 0,000 \\
\hline HSBC & 0,210300 & 0,0405972 & 0,000 \\
\hline Santander & 0,248069 & 0,0500558 & 0,000 \\
\hline AV Villas & 0,084420 & 0,0544723 & 0,123 \\
\hline BCSC & 0,100526 & 0,0509651 & 0,050 \\
\hline Occidente & 0,591529 & 0,0492218 & 0,000 \\
\hline Sudameris & 0,190963 & 0,0395215 & 0,000 \\
\hline $\mathrm{ABN}$ & 2,337891 & 0,8132515 & 0,005 \\
\hline \multicolumn{4}{|c|}{ Variable dependiente: $\operatorname{InY}$} \\
\hline
\end{tabular}

Como el modelo de datos panel es con efectos fijos, entonces se corre por mínimos cuadrados ordinarios (MCO). La prueba a contrastar es una F con tres grados de libertad en el numerador y 164 grados de libertad en el denominador.

Hay dos formas de calcular el estadístico F, las cuales deben dar el mismo resultado. La primera forma es resolver el siguiente contraste de especificación de la prueba F:

$$
F=\frac{(R b-q)^{\prime}\left[R\left(X^{\prime} X\right)^{-1} R^{\prime}\right]^{-1}(R b-q) / J}{e^{\prime} e /(n-K)}
$$

Donde:

$R=\left[\begin{array}{lllllllllllllllllll}0 & 0 & 1 & 0 & 0 & 0 & 0 & 0 & 0 & 0 & 0 & 0 & 0 & 0 & 0 & 0 & 0 & 0 & 0 \\ 0 & 0 & 0 & 1 & 0 & 0 & 0 & 0 & 0 & 0 & 0 & 0 & 0 & 0 & 0 & 0 & 0 & 0 & 0 \\ 0 & 0 & 0 & 0 & 1 & 0 & 0 & 0 & 0 & 0 & 0 & 0 & 0 & 0 & 0 & 0 & 0 & 0 & 0\end{array}\right]$

$q=\left[\begin{array}{l}0 \\ 0 \\ 0\end{array}\right]$

El número de columnas en $\mathrm{R}$ corresponden al número de variables independientes del modelo translogarítmico con efectos fijos, incluyendo a las dicotómicas por banco, el cero de la constante está en la última columna.

\footnotetext{
7 Fuente: Elaboración propia en Stata.
} 
Por un lado, la matriz X incluye todas las variables independientes en el modelo translogarítmico con efectos fijos y, por otro lado, el denominador en (16) es el promedio de los residuos al cuadrado de la regresión del modelo translogarítmico.

Los unos en las columnas 3, 4 y 5 indican que los coeficientes de las variables en las posiciones 3,4 y 5 de la matriz $X$, es decir $\ln ^{2} \mathrm{~K}, \ln ^{2} \mathrm{~L}$ y $\ln \mathrm{K} * \ln \mathrm{L}$ en la tabla 2 son iguales a cero, por ello los ceros en el vector $q$. En esta la constante siempre es la última variable de la regresión, por esto es que el cero de la constante está en la última columna.

El contraste para comparar la función Cobb-Douglas frente a la translogarítmica consiste en plantear en la hipótesis nula que los coeficientes $\alpha_{3}, \alpha_{4}$ y $\alpha_{5}$ de la regresión de la tabla 2 , son todos iguales a cero, frente a la hipótesis alterna de que no son iguales acero.

El planteamiento sería el siguiente:

$$
H_{0}: \alpha_{3}=\alpha_{4}=\alpha_{5}=0
$$

Modelo restringido (2)- Cobb-Douglas

$$
H_{a}: \alpha_{3} \neq \alpha_{4} \neq \alpha_{5} \neq 0
$$

Modelo sin restringir (3)- Translogarítmico

La segunda forma para calcular este mismo estadístico $\mathrm{F}$ es con la siguiente fórmula:

$$
F=\frac{\left(R_{C D}^{2}-R_{T}^{2}\right) / J}{\left(1-R_{C D}^{2}\right) /(n-K)}
$$

Donde $R_{C D}^{2}$ es el $\mathrm{R}$ cuadrado de la regresión con efectos fijos del modelo Cobb-Douglas, que es el modelo restringido en (10) y $R_{T}^{2}$ es el $\mathrm{R}$ cuadrado del modelo Translogarítmico con efectos fijos en, que es el modelo sin restringir en (10).
Por otro lado $\mathrm{J}$ es el número de restricciones, que en este caso son 3, y (n-K) es el número de datos menos el número de regresores en el modelo (10) o en la tabla 2; se excluye la constante.

El estadístico F calculado por (16) o por (18) se presenta en la Tabla 3.

Tabla 3. Contraste de especificación Cobb-Douglas ${ }^{8}$

\begin{tabular}{|c|c|c|}
\hline & Estadístico & Probabilidad \\
\hline Prueba F & 2,87 & 0,0380 \\
\hline
\end{tabular}

Los resultados de la Tabla 3 indican que se aprueba la hipótesis alterna, es decir, el modelo correcto es el translogarítmico. Si los coeficientes no son iguales a cero, entonces el modelo (10) es el correcto, es decir, el modelo no restringido.

\subsection{Elasticidades}

A partir de los datos de la Tabla 2 se deducen las elasticidades del capital y del trabajo, según las fórmulas (4) y (5).

Las elasticidades del capital y del trabajo son:

$$
\begin{gathered}
\varepsilon_{K}=0,9290 \\
\varepsilon_{L}=-0,01681
\end{gathered}
$$

Lo que indican las elasticidades es que los préstamos bancarios tienen una elasticidad cercana a uno, es decir, por cada uno por ciento que aumentan los préstamos bancarios entonces los ingresos totales de los bancos aumentan un 0,92\%. Los aumentos del trabajo no tienen un efecto significativo sobre los ingresos del sector financiero.

\subsection{Productividades marginales}

Las productividades marginales del capital, del trabajo y las cruzadas, según (6), (7) y (8) son respectivamente:

8 Fuente: Elaboración propia en Stata. 


$$
\begin{gathered}
\varepsilon_{K K}=-0,074 \\
\varepsilon_{L L}=-0,063 \\
\varepsilon_{K L}=\varepsilon_{L K}=0,079
\end{gathered}
$$

Lo que indica que existen productividades marginales decrecientes. A medida que aumentan, tanto los préstamos como el trabajo, entonces sus productividades disminuyen.

El hecho de que las segundas derivadas cruzadas sean positivas indica que el capital y el trabajo en el sector financiero son complementarios.

\subsection{Significancia de las elasticidades, elasticidades unitarias y elasticidad de escala}

Para hacer un análisis un poco más detallado de las elasticidades hay que encontrar los errores estándar de las mismas.

Estadísticamente se calculan los errores estándar de las elasticidades con las raíces cuadradas de las varianzas de las elasticidades del capital y el trabajo, según las siguientes fórmulas:

$$
\begin{aligned}
& \operatorname{Var}\left[\varepsilon_{\mathrm{K}}\right]=\operatorname{VarEst}\left[\alpha_{1}+\alpha_{3} \overline{\ln K}+\alpha_{5} \overline{\ln L}\right]=w K(\operatorname{VarEst}[\mathrm{b}]) \mathrm{wK}^{\prime} \\
& \operatorname{Var}\left[\varepsilon_{\mathrm{L}}\right]=\operatorname{VarEst}\left[\alpha_{2}+\alpha_{4} \overline{\ln \mathrm{L}}+\alpha_{5} \overline{\ln K}\right]=\mathrm{wL}(\operatorname{Var} E s t[\mathrm{~b}]) \mathrm{wL}^{\prime}
\end{aligned}
$$

Donde:

$$
\begin{gathered}
w K=(1,0, \overline{\ln K}, 0, \overline{\ln L}, 0) \\
w L=(0,1,0, \overline{\ln L}, \overline{\ln K}, 0)
\end{gathered}
$$

El resultado de (19) y (20) son escalares. Además Var.Est[b] es la matriz de varianzas y covarianzas de la regresión (10), es decir, la translogarítmica. Por otro lado, $\overline{\ln K}$ es el promedio de los logaritmos del capital y $\overline{\ln L}$ es el promedio de los logaritmos del trabajo.

Los resultados de la Tabla 4 indican que la elasticidad del capital es altamente significativa, pero la elasticidad del trabajo es igual a cero.

Para realizar la prueba de elasticidad unitaria del capital se calcula el siguiente estadístico F:

$$
F=\frac{\left(\varepsilon_{K}-1\right)^{2}}{\operatorname{Var}\left[\varepsilon_{K}\right]}
$$

Las hipótesis son las siguientes:

$$
\begin{aligned}
& H_{0}: \varepsilon_{\mathrm{K}}=1 \\
& H_{a}: \varepsilon_{\mathrm{K}} \neq 1
\end{aligned}
$$

El resultado en (20) indica que la F calculada es igual a 2,87 . La $\mathrm{F}$ de la tabla con una probabilidad de 0,95 , con un grado de libertad en el numerador $y$ 164 grados de libertad en el denominador es igual a 3,84. Lo que indica que el $\mathrm{F}$ calculado es menor que el F de la tabla, por lo que se aprueba la hipótesis nula, es decir, se puede decir que la elasticidad del capital es unitaria. Por cada uno por ciento que aumenta el capital entonces el output aumenta en un uno por ciento.

Por último la elasticidad a escala es igual a la suma de las elasticidades del capital y del trabajo, por lo tanto es:

Tabla 4. Significancia de las elasticidades-modelo translogarítmico ${ }^{9}$

\begin{tabular}{|c|c|c|c|c|}
\hline & Elasticidades & Error Estándar & Estadístico T & Probabilidad \\
\hline Capital & 0,92906 & 0,03133546 & 29,64886 & $2,37 \mathrm{E}-67$ \\
\hline Trabajo & $-0,01681$ & 0,04667857 & $-0,36030118$ & 0,71905394 \\
\hline
\end{tabular}

9 Fuente: Elaboración propia. 


$$
\varepsilon_{\text {esc }}=\varepsilon_{K}+\varepsilon_{L}=0,91225
$$

Lo que indica que por cada uno por ciento que aumenta tanto el capital como el trabajo entonces el ingreso total aumenta un 0,91 por ciento.

\section{Conclusiones}

El principal objetivo de este trabajo es explicar en forma pormenorizada el desarrollo matemático y econométrico al comparar las funciones de producción Cobb-Douglas y la translogarítmica, en el sector financiero colombiano.

Se encuentra que la función de producción matemática que mejor se ajusta a los datos del sector financiero en Colombia es la translogarítmica frente a la Cobb-Douglas, lo que implica que las elasticidades no son constantes para la muestra tomada. Econométricamente, al utilizar la metodología de datos panel, el modelo que se debe correr es el de efectos fijos, frente al modelo sin efectos o al de efectos aleatorios, lo que indica que existen diferencias significativas en los retornos del capital y del trabajo entre bancos.

De lo anterior se puede afirmar que los bancos en Colombia tienen diferentes productividades, siendo el más eficiente el banco de Occidente y el menos eficiente el Amro Bank, en cuanto a los retornos del capital y del trabajo sobre el output, que son los ingresos de los mismos.

Un análisis de los resultados indica que la elasticidad del capital es altamente significativa y, dado que el negocio de los bancos parte de los préstamos, entonces no es de extrañar este resultado. Pero, por otro lado, la elasticidad del trabajo no es significativa, lo que indica que el factor laboral en el sector financiero colombiano es solo un instrumento que facilita el negocio de intermediación financiera, pero no es un factor determinante para el negocio.

Lo anterior también se explica por el hecho de que la productividad cruzada entre capital y trabajo es positiva, por lo que se infiere que estos dos factores son complementarios. Este resultado es lógico, dado que en el sector financiero los trabajadores complementan los préstamos; es decir, para que existan préstamos es necesario que existan los trabajadores que los incentivan y los promueven. Los trabajadores en ningún caso son sustitutos de los préstamos.

Esto corrobora los resultados con las elasticidades, el trabajo complementa al capital en los bancos comerciales, pero en ningún momento lo sustituye.

Se demuestra también que existen rendimientos decrecientes, de lo cual se deduce que el aumento del trabajo y del capital no necesariamente mejora los retornos de los mismos, en cuanto a productividad se refiere.

La elasticidad del factor capital es igual a uno, por lo que un aumento de un uno por ciento del capital genera un aumento de un uno por ciento de los ingresos bancarios. Este resultado es apenas lógico por el tipo de negocio de los bancos.

\section{Referencias}

Arrow, K., Chenery, H., Minhas, B. \& Solow, R. (1961). Capitallabor substitution and economics efficiency. En: The Review of Economics and Statistics, 43(43).

Brown, B. (1970) Tests for Cobb-Douglas and CES production functions. En: International Economic Review, 11(1): 77-83.

Burras, D. \& Moroney, J. (1975). Substitution among capital, labor, and natural resource products in American manufacturing. En: Journal of Political Economy, 83(1): 57-82.

Christensen, L., Jorgenson, D. \& Lau, L. (1973). Transcendental logarithmic production frontiers. En : The Review of Economics and Statistics, 55(1).

Estrada, D. \& Osorio, P. (2004). Efectos del Capital financiero en la Eficiencia del Sistema Bancario Colombiano. Ensayos sobre política económica, Banco de la República.

Fletcher, L. \& Lu, Y. (1968). A generalization of the CES production function. En: The Review of Economics and Statistics, 50(4).

Greene, W. (2001). Análisis econométrico. Capítulo 14. Editorial Prentice Hall. New Jersey.

Humprey, T. (2001). Algebraic production functions and their uses before Cobb-Douglas. Federal Reserve Bank of Richmond Economic Quarterly. Washington.

Kim, H. (1992). The translog production function and variable returns to scale. En: The Review of Economics and Statistics, 74(3): 546-552.

Klump, R. \& Preissler, H. (2000). CES Production Functions and Economic Growth. En: The Scandinavian Journal of Economics, 102(1): 41-56.

Kraipornsak, P. (2001). ¿What different ist the production function estimated by different methods? An empirical estimation comparison. Faculty of Economics, Chulanlongkorn University. 
Mefford, R. (1986). Introducing management into the production function. En: The Review of Economics and Statistics, 68(1): 96-104.

Ryu, H. \& Zellner, A. (1998). Alternative functional forms for production, cost and returns to scale function. En: Journal of Applied Econometrics, 13.
Server, R. \& Melián, A. (2001). Evaluación de la eficiencia de las entidades financieras en las secciones de crédito de las cooperativas. CIRIEC-España.

Walters, A. (1963). Production functions and cost functions: and econometric survey. En: Econométrica, 31. 


\section{ANEXOS}

Anexo 1. Regresión sin efectos ${ }^{1}$

\begin{tabular}{r|crc} 
Source & SS & df & MS \\
\hline $\begin{array}{r}\text { Model } \\
\text { Residual }\end{array}$ & 195.089798 & 5 & 39.0179596 \\
& 3.28291459 & 176 & .018652924 \\
\hline Total & 198.372712 & 181 & 1.09598184
\end{tabular}

Number of obs $=182$

F( $5, \quad 176)=2091.79$

Prob > $\mathbf{F}=0.0000$

R-squared $=0.9835$

Adj R-squared $=0.9830$

Root MSE $=.13658$

\begin{tabular}{r|rrrrrr}
\hline Inpro & Coef. & Std. Err. & $\mathrm{t}$ & $\mathrm{p}\rangle|\mathrm{t}|$ & [958 Conf. Interval] \\
& & & & & & \\
\hline Incap & 1.84228 & .3054695 & 6.03 & 0.000 & 1.239425 & 2.445134 \\
Intra & -.6986523 & .3358653 & -2.08 & 0.039 & -1.361494 & -.0358106 \\
Incap2 & -.1494379 & .0552149 & -2.71 & 0.007 & -.2584063 & -.0404694 \\
Intra2 & -.0089042 & .0628242 & -0.14 & 0.887 & -.1328898 & .1150815 \\
Incata & .0750057 & .0521712 & 1.44 & 0.152 & -.0279558 & .1779673 \\
_cons & -.7339373 & .9538829 & -0.77 & 0.443 & -2.616458 & 1.148583 \\
\hline
\end{tabular}

Anexo 2. Regresión con efectos fijos ${ }^{2}$

\begin{tabular}{|c|c|c|c|c|c|c|}
\hline \multirow{2}{*}{$\begin{array}{l}\text { i. empresa } \\
\text { Source }\end{array}$} & \multicolumn{2}{|c|}{ _Iempresa_1-13 } & \multicolumn{2}{|c|}{ (naturally coded; } & \multicolumn{2}{|c|}{; _Iempresa_1 omitted) } \\
\hline & ss & df & MS & & Number of obs & 182 \\
\hline Model & 197.362345 & 1711. & 95497 & & Prob $>$ F & $=0.0000$ \\
\hline Residual & 1.01036765 & 164.00 & 60778 & & $\mathrm{R}$-squared & -0.9949 \\
\hline Total & 198.372712 & $181 \quad 1.0$ & 98184 & & Root MSE & $=.07849$ \\
\hline Inpro & Coef. & Std. Err. & $\mathrm{t}$ & $p>|t|$ & [958 Conf. & Interval] \\
\hline Incap & .9583728 & .2251422 & 4.26 & 0.000 & .5138218 & 1.402924 \\
\hline Intra & -.2743475 & .2163843 & -1.27 & 0.207 & -.7016056 & .1529107 \\
\hline 1ncap2 & -.0749739 & .0375462 & -2.00 & 17 & -.1491103 & -.0008376 \\
\hline Intra2 & -.0638088 & .0418219 & -1.53 & 9 & -.1463876 & .0187699 \\
\hline Incata & .0793938 & .0345116 & 2.30 & 3 & .0112494 & .1475382 \\
\hline Iempresa_2 & .32040 & .0556479 & 5.76 & 0 & .2105229 & .4302805 \\
\hline Iempresa 3 & .28 & .052 & 17 & & .18 & .3926225 \\
\hline Iempresa 4 & .18 & .04 & 9 & & 2 & .2767437 \\
\hline Iempresa_ 5 & .259 & .0520588 & 4.98 & & 26 & .3620163 \\
\hline Iempresa 6 & .3447 & .0539656 & 6.39 & 0.0 & .2381815 & .4512952 \\
\hline _Iempresa_ 7 & .2566668 & .0515412 & 4.98 & 0.000 & .154897 & .3584366 \\
\hline Iempresa_8 & .2103002 & .0405972 & 5.18 & 0.000 & .1301396 & .2904608 \\
\hline Iempresa_ 9 & .2480692 & .0500558 & 4.96 & 0.000 & .1492323 & .3469061 \\
\hline _empresa_10 & .0844195 & .0544723 & 1.55 & 0.123 & -.023138 & .1919769 \\
\hline Iempresa_11 & .1005257 & .0509651 & 1.97 & 0.050 & -.0001067 & .201158 \\
\hline Iempresa_12 & .5915293 & .0492218 & 12.02 & 0.000 & .4943391 & .6887194 \\
\hline Iempresa_13 & .1909625 & .0395215 & 4.83 & 0.000 & .1129259 & .2689991 \\
\hline cons & 2.337891 & .8132515 & 2.87 & 0.005 & .7320982 & 3.943685 \\
\hline
\end{tabular}

Fuente: Elaboración propia.

Fuente: Elaboración propia. 
Anexo 3. Regresión con efectos aleatorios ${ }^{3}$

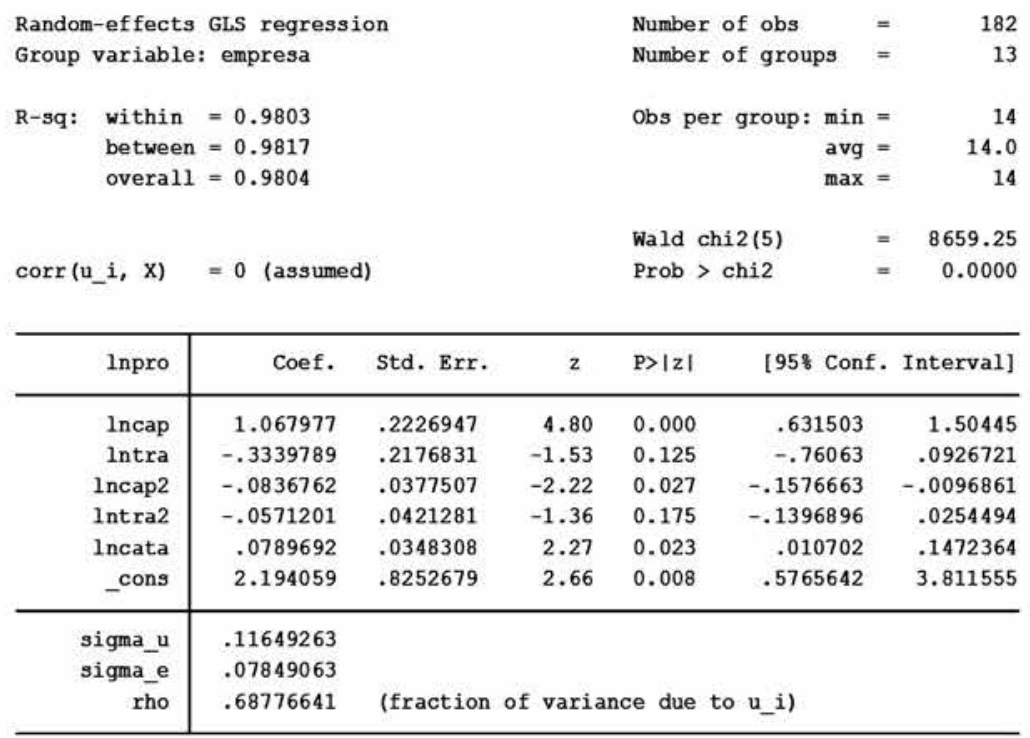

Anexo 4. Prueba F: efectos fijos versus $\sin$ efectos $^{4}$

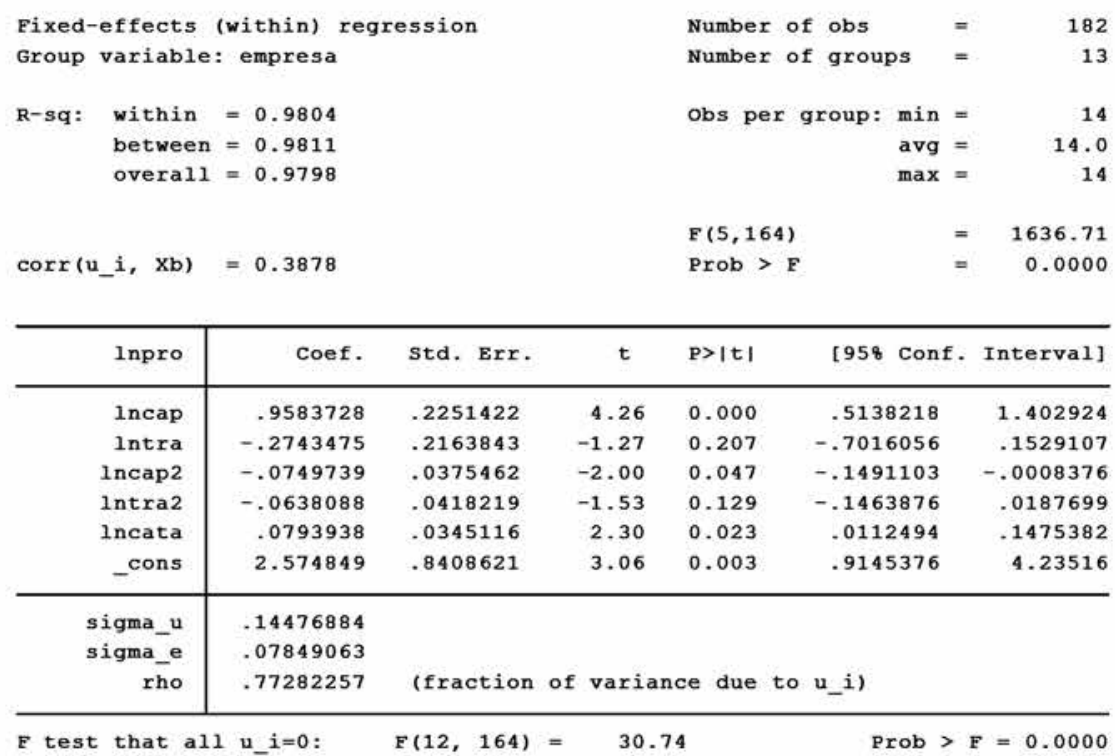

3 Fuente: Elaboración propia.

4 Fuente: Elaboración propia. 
Anexo 5. Prueba de Breusch-Pagan ${ }^{5}$

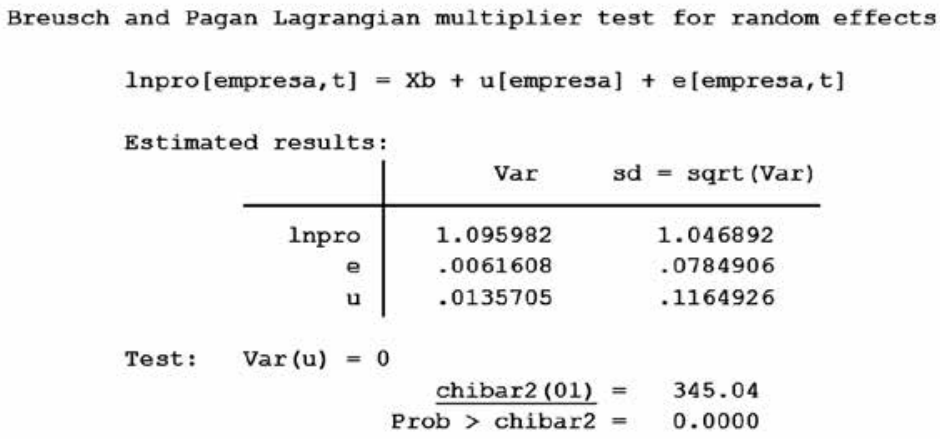

Anexo 6. Prueba de Hausman ${ }^{6}$

\begin{tabular}{r|rrrr} 
& \multicolumn{1}{c}{$\begin{array}{c}\text { (b) } \\
\text { efefi1 }\end{array}$} & $\begin{array}{c}\text { Coefficients } \\
\text { efeale1 }\end{array}$ & $\begin{array}{c}\text { (b-B) } \\
\text { Difference }\end{array}$ & $\begin{array}{c}\text { sqrt (diag (V_b-V_B)) } \\
\text { S.E. }\end{array}$ \\
\hline Incap & .9583728 & 1.067977 & -.1096037 & .033107 \\
Intra & -.2743475 & -.3339789 & .0596315 &. \\
Incap2 & -.0749739 & -.0836762 & .0087022 &. \\
1ntra2 & -.0638088 & -.0571201 & -.0066887 &. \\
1ncata & .0793938 & .0789692 & .0004246 &. \\
\hline
\end{tabular}

b = consistent under Ho and Ha; obtained from regress B - inconsistent under $\mathrm{Ha}$, efficient under $\mathrm{HO}$; obtained from xtreg

Test: Ho: difference in coefficients not systematic

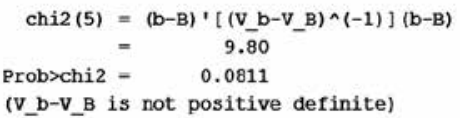

Anexo 7. Prueba F para contraste modelo restringido versus modelo sin restringir ${ }^{7}$

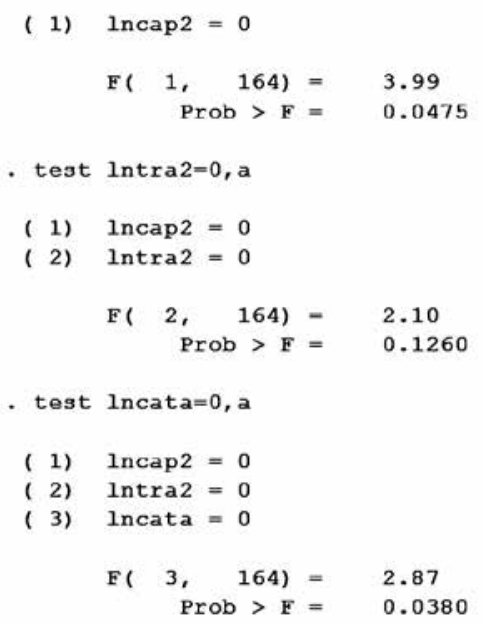

Fuente: Elaboración propia. Fuente: Elaboración propia. Fuente: Elaboración propia. 